\title{
Behavioral Analysis of Judges in Subjective Evaluation Based on Statistics
}

\author{
Pei Xuesheng ${ }^{1,2}$ Zheng Xiwen²
}

1 School of Mechatronic Engineering and Automation, Shanghai University, Shanghai 200072

2 School of Art and Design, Henan University of Science and Technology, Luoyang 471023

Keywords: subjective evaluation, statistical analysis, evaluation criterion, judges

\begin{abstract}
In this paper, a comprehensive evaluation method of judges' subjective judgment is presented. Problems in evaluating art work were analyzed by statistical tools which were also used to analyze the scores achieved by the subjective evaluation of judges while also evaluating the fairness, level, and criterion of the judges. Advantages and disadvantages of the evaluation methods were also given here. The assessment results will provide a reference for judge selection for a review organization in the future.
\end{abstract}

\section{Introduction}

Subjective evaluation is indispensable in people's life and their work. Especially, in the field of art, the evaluation of artistic work is characterized by uncertainty, imprecision, and subjectivity. ${ }^{[1]}$ For subjective evaluation, it is common practice for several evaluators to evaluate an objective object. This method is simple and practicable, and each judge will give an evaluation from one of the several aspects of an object in his own perspective before reaching a comprehensive score. Before collecting statistical results, two extreme scores were removed and the remaining scores were averaged to get the resultant score of the object. There was inconsistency between the scores given by different judges and this is caused by their strictness with the criterion and the consistency of the judges. ${ }^{[2][3]}$

\section{Problems with subjective evaluation}

\subsection{Judge's strictness with the criterion}

In evaluation, a judge's strictness with the criterion may be different and this will lead to varied scores and relatively greater error in statistical findings.

a. Greater numbers will dominate lesser ones.

For example, in evaluating height, three different units of "meter", "decimeter", and "centimeter" are used by the judges. If height values represented in different units are aggregated for the average value, the greater numbers will dominate the lesser ones. This will make the evaluation of the numbers in "meter" invalid. Similarly, evaluation with greater differentials will predominate those with lesser differentials.

b. Removing two extreme data.

As for judges with loose or strict adherence to criterion of the evaluation, their evaluation results may be invalidated due to greater deviation which can be misunderstood as unfairness. Thus, it's unfair to remove the two extreme scores without any treatment. ${ }^{[4]}$ In actual practice, evaluation of such judges can be the most informed.

\subsection{Impartiality of judges}

Judges can arrive at partial evaluation due to interests, guanxi, or other factors. Subjective evaluations can be greatly influenced by the fairness of the judge and such evaluation results are often questioned. 


\subsection{Professionalism of the judges}

Judges are usually selected from experts or leaders in a certain field. Inevitably, some unsatisfactory judges maybe selected in this process. Evaluation results given by such judges often deviate from the true picture.

\subsection{Weight problem}

Judges provide evaluation results on the basis of their knowledge and experience, so subjectivity of the judges should be minimized. ${ }^{[5]}$ Sometimes, each judge will give their careful evaluation of all aspects of one piece of work in the hope of offering more accurate results. However, the significance of each aspect considered in the evaluation is different. As a result, evaluation by different judges of the same work may vary greatly and unfair evaluation is seen on occasion. Such evaluation results may be questioned.

\section{Data processing}

Reliability of this evaluation is analyzed here. Given an evaluation, there are pieces of work $A_{1}$ $A_{2} \ldots A_{n}$, and judges $B_{1} B_{2} \ldots B_{m}$. The evaluation matrix is given as:

$$
\mathrm{AB}=\left[\begin{array}{ccc}
\mathrm{A} 1 \mathrm{~B} 1 & \cdots & \mathrm{AnB} 1 \\
\vdots & \ddots & \vdots \\
\mathrm{A} 1 \mathrm{Bm} & \cdots & \mathrm{AnBm}
\end{array}\right]
$$

For this matrix, the scoring result of the piece $\mathrm{A}_{\mathrm{i}}$ is generally achieved by the average:

$$
\mathrm{Ai}=\left(\sum_{\mathrm{j}=1}^{\mathrm{m}} \mathrm{AiBj}-\max A \mathrm{~B} \mathrm{j}-\min A \mathrm{~B} B \mathrm{j}\right) /(\mathrm{m}-2) .
$$

Possible problems of this method have been discussed in the previous section. Here, we propose a new statistical method for calculation.

\subsection{To begin, the evaluation scale coefficient $S_{j}$ of each judge must be determined.}

Create an evaluation scale coefficient matrix

$$
B j=\frac{\sum_{i=1}^{n} A i B j}{n}, B=\frac{\sum_{j=1}^{m} B j}{m}, S j=\frac{B}{B j} .
$$

$$
S=\left[\begin{array}{ccc}
S 1 & \cdots & 0 \\
\vdots & \ddots & \vdots \\
0 & \cdots & \text { Sm }
\end{array}\right]
$$

Next, multiply the evaluation scale coefficient matrix $S$ and evaluation matrix $A B$ to achieve the new evaluation matrix

$\mathrm{AB}^{\prime}=\mathrm{S}^{*} \mathrm{AB}$

The new scoring result of the piece Ai is generally achieved by the average:

$$
\mathrm{Ai}^{\prime}=\left(\sum_{\mathrm{j}=1}^{\mathrm{m}} \mathrm{AiBj}-\operatorname{maxAiBj} \mathrm{j}^{\prime}-\min A \mathrm{ABj} \mathrm{j}^{\prime}\right) /(\mathrm{m}-2) .
$$

\subsection{Behavioral analysis of judges}

Standard deviation of work $\mathrm{Ai}$ is evaluation results

$$
\delta i=\sqrt{\frac{1}{m} \sum_{j=1}^{m}(A i B j-A i)^{\wedge} 2}
$$

Deviation coefficient Csij of evaluation results.

Deviation coefficient of judge $\mathrm{Bj}$

$$
\mathrm{Csij}=(\mathrm{AiBj}-\mathrm{Ai}) / \delta \mathrm{i}
$$

$$
\begin{gathered}
C B j=\frac{1}{n} \sum_{i=1}^{n} C s i j \\
B \delta j=\sqrt{\frac{1}{n} \sum_{i=1}^{n}(C s i j-C B j)^{\wedge} 2}
\end{gathered}
$$

Evaluation behavior of the judge may be achieved by the judge's scale coefficient $S_{j}$ and 
Deviation coefficient $\mathrm{CBj}$ and the standard deviation $\mathrm{B} \delta \mathrm{j}$ of the evaluation results.

$\mathrm{S}_{\mathrm{j}}<1$ or $\mathrm{CBj}>0$ denotes that judge $\mathrm{Bj}$ is less strict, and $\mathrm{S}_{\mathrm{j}}>1$ or $\mathrm{CBj}<0$ denotes that judge $\mathrm{Bj}$ is more strict.

Less $B \delta j$ indicates consistency between the judge's evaluation and the results. Greater $B \delta j$ indicates inconsistency between the judge's evaluation and the results which may be a result of the judge's less experience of professionalism. A removed evaluation may reflect extreme judgment of any judge.

The above results can be automatically analyzed by a computer program. Enter the number of pieces of work and the number of judges, as well as enter the judges' judgment of each piece of work. Afterwards, the computer will automatically analyze the evaluation of each judge and produce its views for reference.

\subsection{A difference correction method can be used for the judges' evaluation:}

To start, calculate the maximum difference between all of the evaluation results of the judges:

$$
\mathrm{dj}=\operatorname{maxAiBj}-\min A i B j
$$

Determine the range of evaluation[a, $a+b]$; performa normalized process of the original data in order to determine the new evaluation matrix:

$$
\begin{gathered}
A i B j "=a+b * \frac{A i B j-\min A i B j}{d j} \\
A B "=\left[\begin{array}{ccc}
A 1 B 1 " & \ldots & \text { AnB1" } \\
\vdots & \ddots & \vdots \\
A 1 B m " & \cdots & \text { AnBm" }
\end{array}\right]
\end{gathered}
$$

Afterwards, use this evaluation matrix to calculate the evaluation results of each piece of work before placing their ranks.

\section{Analysis of cases}

The following table shows the original data of the evaluated pieces of work.

Table 1 The original data

\begin{tabular}{|c|c|c|c|c|c|}
\hline & Judge 1 & Judge 2 & Judge 3 & Judge 4 & Judge 5 \\
\hline Work 1 & 98 & 89 & 92 & 92 & 88 \\
\hline Work 2 & 93 & 87 & 92 & 90 & 93 \\
\hline Work 3 & 91 & 85 & 87 & 88 & 78 \\
\hline Work 4 & 89 & 83 & 87 & 86 & 86 \\
\hline Work 5 & 87 & 81 & 85 & 84 & 85 \\
\hline
\end{tabular}

Table 2 Average \& rank

\begin{tabular}{|c|r|r|r|c|}
\hline & \multicolumn{1}{|c|}{$\begin{array}{c}\text { Direct } \\
\text { Average } \\
\text { score }\end{array}$} & Rank & $\begin{array}{c}\text { Average score gained by } \\
\text { removing the two limits }\end{array}$ & Rank \\
\hline Work 1 & 91.8 & 1 & 91 & 2 \\
\hline Work 2 & 91 & 2 & 91.66667 & 1 \\
\hline Work 3 & 85.8 & 4 & 86.66667 & 3 \\
\hline Work 4 & 86.2 & 3 & 86.33333 & 4 \\
\hline Work 5 & 84.4 & 5 & 84.66667 & 5 \\
\hline
\end{tabular}

Table 3 Evaluation scale factor

\begin{tabular}{|c|c|c|c|c|c|}
\hline & Judge 1 & Judge 2 & Judge 3 & Judge 4 & Judge 5 \\
\hline $\mathrm{S}$ & 0.958952 & 1.033412 & 0.991422 & 0.998182 & 1.021395 \\
\hline
\end{tabular}


Table 4 Multiply the evaluation scale and the original data

\begin{tabular}{|r|r|r|r|r|r|}
\hline & \multicolumn{1}{|c|}{ Judge 1 } & \multicolumn{1}{c|}{ Judge 2 } & \multicolumn{1}{c|}{ Judge 3 } & \multicolumn{1}{c|}{ Judge 4 } & \multicolumn{1}{c|}{ Judge 5 } \\
\hline Work 1 & 93.97729 & 91.97365 & 91.21084 & 91.83273 & 89.88279 \\
\hline Work 2 & 89.18253 & 89.90682 & 91.21084 & 89.83636 & 94.98977 \\
\hline Work 3 & 87.26463 & 87.84 & 86.25372 & 87.84 & 79.66884 \\
\hline Work 4 & 85.34672 & 85.77318 & 86.25372 & 85.84364 & 87.84 \\
\hline Work 5 & 83.42882 & 83.70635 & 84.27088 & 83.84727 & 86.8186 \\
\hline
\end{tabular}

Table 5 New average \& rank

\begin{tabular}{|c|c|c|r|c|}
\hline & $\begin{array}{c}\text { Direct } \\
\text { Average } \\
\text { score }\end{array}$ & Rank & \multicolumn{1}{|c|}{$\begin{array}{c}\text { Average score gained } \\
\text { by removing the two } \\
\text { limits }\end{array}$} & Rank \\
\hline Work 1 & 91.77546 & 1 & 91.6724 & 1 \\
\hline Work 2 & 91.02526 & 2 & 90.31801 & 2 \\
\hline Work 3 & 85.77344 & 4 & 87.11945 & 3 \\
\hline Work 4 & 86.21145 & 3 & 85.95685 & 4 \\
\hline Work 5 & 84.41439 & 5 & 83.9415 & 5 \\
\hline
\end{tabular}

Table 6 Standard deviation and deviation coefficient

\begin{tabular}{|c|r|r|r|r|r|}
\hline$\delta \mathrm{i}$ & \multicolumn{1}{|c|}{ Csi1 } & \multicolumn{1}{c|}{ Csi2 } & \multicolumn{1}{c|}{ Csi3 } & \multicolumn{1}{c|}{ Csi4 } & \multicolumn{1}{c|}{ Csi5 } \\
\hline 3.487119 & 1.777972 & -0.80296 & 0.057354 & 0.057354 & -1.08972 \\
\hline 2.280351 & 0.877058 & -1.75412 & 0.438529 & -0.43853 & 0.877058 \\
\hline 4.354308 & 1.19422 & -0.18373 & 0.275589 & 0.505247 & -1.79133 \\
\hline 1.939072 & 1.44399 & -1.65027 & 0.412568 & -0.10314 & -0.10314 \\
\hline 1.959592 & 1.326807 & -1.73506 & 0.306186 & -0.20412 & 0.306186 \\
\hline
\end{tabular}

Table 7 Behavioral analysis of judges

\begin{tabular}{|c|r|r|r|r|r|}
\hline & Judge 1 & Judge 2 & Judge 3 & Judge 4 & Judge 5 \\
\hline $\mathrm{CBj}$ & 1.324009 & -1.22523 & 0.298045 & -0.03664 & -0.36019 \\
\hline $\mathrm{B} \delta \mathrm{j}$ & 1.0997 & 1.165085 & 0.274085 & 0.316468 & 1.003639 \\
\hline Conclusion & Loose & Strict & General & Consistency & Inconsistency \\
\hline
\end{tabular}

\subsection{A difference correction method}

Table 8 The maximum difference of Judge

\begin{tabular}{|c|c|c|c|c|c|}
\hline & Judge 1 & Judge 2 & Judge 3 & Judge 4 & Judge 5 \\
\hline $\mathrm{dj}$ & 11 & 8 & 7 & 8 & 15 \\
\hline
\end{tabular}

Table 9 when $\mathrm{a}=60, \mathrm{~b}=40$

\begin{tabular}{|c|r|r|r|r|r|r|r|}
\hline & Judge1 & Judge & Judge3 & Judge4 & Judge5 & $\begin{array}{c}\text { Average score } \\
\text { gained by } \\
\text { removing the two } \\
\text { limits }\end{array}$ & Rank \\
\hline Work1 & 100 & 100 & 100 & 100 & 86.67 & 100 & 1 \\
\hline Work2 & 81.82 & 90 & 100 & 90 & 100 & 93.333 & 2 \\
\hline Work3 & 74.55 & 80 & 71.43 & 80 & 60 & 75.325 & 3 \\
\hline Work4 & 67.27 & 70 & 71.43 & 70 & 81.33 & 70.476 & 4 \\
\hline Work 5 & 60 & 60 & 60 & 60 & 78.67 & 60 & 5 \\
\hline
\end{tabular}

\section{Conclusion}

The actual case shows that the judges of different types judged the works at the same scale by increasing the scale factor of the judges, which makes the evaluation more objective and accurate. In addition, statistical tools which were used to analyze the scores achieved by the subjective 
evaluation of judges while also evaluating the fairness, level, and criterion of the judges. The assessment results will provide a reference for judge selection for a review organization in the future.

Pei Xuesheng: (1969,12-) Shangqiu, Henan, China, Associate professor, Doctoral candidate, Research interests: Industrial design methods and applications, Product digital design etc.

\section{Reference}

[1] Antonsson E K, Cagan J. Formal engineering design synthesis[M]. New York: Cambridge University Press, 2001

[2] Li Yong, Wang Yougui. Evaluation of the Judges: A New Statistic and Evaluation Method[J]. Statistics and Decision. 2014(18);

[3] LVShu-long, LIANGFei-bao, LIUWen-li. An evaluation model of rater score [J] Journal of Fuzhou University (Natural Science )Vol.38 No. 3 Jun .2010

[4] Xia Jinyang, Research of Statistics on "Invalid Judge" in Sports Teaching Competition [J], Physical Education, 2012 (11).

[5] Fan Zhongguang, Evaluation of Third-party Logistics Service Provider Based on Three-parameter Interval Number Model[J], Logistics Technology (Equipment), 2015 (1) 